\title{
Multicentric assessment of the efficacy and tolerability of dihydroartemisinin-piperaquine compared to artemether-lumefantrine in the treatment of uncomplicated Plasmodium falciparum malaria in sub-Saharan Africa
}

William Yavo ${ }^{1,6^{*}}$, Babacar Faye ${ }^{2}$, Thomas Kuete ${ }^{3}$, Vincent Djohan', Serge A Oga ${ }^{4}$, Richard R Kassi ${ }^{5}$, Mariama Diatta ${ }^{2}$, Moor V Ama ${ }^{3}$, Roger Tine ${ }^{2}$, Jean-Louis Ndiaye ${ }^{2}$, Jean-Bedel Evi', Albert Same-Ekobo ${ }^{3,7}$, Oumar Faye ${ }^{2}$ and Moussa Koné ${ }^{1}$

\begin{abstract}
Background: The choice of appropriate artemisinin-based combination therapy depends on several factors (cost, efficacy, safety, reinfection rate and simplicity of administration). To assess whether the combination dihydroartemisinin-piperaquine (DP) could be an alternative to artemether-lumefantrine (AL), the efficacy and the tolerability of the two products for the treatment of uncomplicated falciparum malaria in sub-Saharan Africa have been compared.
\end{abstract}

Methods: A multicentric open randomized controlled clinical trial of three-day treatment of DP against AL for the treatment of two parallel groups of patients aged two years and above and suffering from uncomplicated falciparum malaria was carried out in Cameroon, Côte d'Ivoire and Senegal. Within each group, patients were randomly assigned supervised treatment. DP was given once a day for three days and AL twice a day for three days. Follow-up visits were performed on day 1 to 4 and on day 7, 14, 21, 28 to evaluate clinical and parasitological results. The primary endpoint was the recovery rate by day 28 .

Results: Of 384 patients enrolled, 197 were assigned DP and 187 AL. The recovery rates adjusted by genotyping, $99.5 \%$ in the DP group and $98.9 \%$ in the AL group, were not statistically different ( $p=0.538$ ). No Early Therapeutic Failure (ETF) was observed. At day 28, two patients in the DP group and five in AL group had recurrent parasitaemia with Plasmodium falciparum. In the DP group, after PCR genotyping, one of the two recurrences was classified as a new infection and the other as recrudescence. In AL group, two recurrences were classified after correction by PCR as recrudescence. All cases of recrudescence were classified as Late Parasitological Failure (LPF). In each group, a rapid recovery from fever and parasitaemia was noticed. More than $90 \%$ of patients did no longer present fever or parasitaemia 48 hours after treatment. Both drugs were well tolerated. Indeed, no serious adverse events were reported during the follow-up period. Most of the adverse events which developed were moderate and did not result in the treatment being stopped in either treatment group.

Conclusions: Dihydroartemisinin-piperaquine was as effective and well-tolerated as artemether-lumefantrine in the treatment of uncomplicated falciparum malaria. In addition, dihydroartemisinin-piperaquine, a single daily dose, could be an advantage over artemether-lumefantrine in Africa because of better treatment observance.

\footnotetext{
* Correspondence: yavowilliam@yahoo.fr

'Department of Parasitology and Mycology, Faculty of Pharmaceutical and Biological Sciences, Abidjan, Côte d'Ivoire

Full list of author information is available at the end of the article
}

(c) 2011 Yavo et al; licensee BioMed Central Ltd. This is an Open Access article distributed under the terms of the Creative Commons 


\section{Background}

Malaria caused by Plasmodium falciparum is a serious concern for public health and development in Africa. The most part of the continent is facing increasing resistance of this parasite to chloroquine and sulphadoxine-pyrimethamine, the widely available and cheap antimalarial drugs. In order to overcome this resistance problem, several African countries have recently adopted artemisinin-based combination therapy (ACT) as firstline treatment for uncomplicated malaria $[1,2]$. The artemether-lumefantrine (AL) combination proved to be highly effective and well-tolerated in several studies in Africa [3-5]. However, a twice daily dose schedule of AL and its need to be administered together with a fat-rich meal [6] are a disadvantage.

Dihydroartemisinin-piperaquine (DP) is a new ACT administered as single daily dose that has proved to be well tolerated and highly effective against uncomplicated falciparum malaria in southeast Asia [7-12] and in eastern Africa [13,14]. However, no clinical trial concerning this anti-malarial drug has yet been conducted in western and central Africa. Therefore, a randomized non-inferiority open trial has been carried out to compare efficacy and tolerability of DP to that of AL, the reference ACT, in the treatment of uncomplicated falciparum malaria in Cameroon, Côte d'Ivoire and Sénégal. The hypothesis was that DP is as efficacious and safe as AL.

\section{Methods}

\section{Study site and population}

This study was carried out from November 2006 to May 2008 in three sub-Saharan countries: Senegal and Côte d'Ivoire are in western Africa, Cameroon is in central Africa. In Cameroon, the study took place at the University Hospital Centre. In Côte d'Ivoire, it took place at the health care centre of Bocabo, which is located in Abobo in the north of Abidjan (the economic capital). In Senegal, the assessment took place at the health care centre of Darou Marnane in the sanitary district of Touba, which is located in the centre of the country at $200 \mathrm{~km}$ of Dakar.

The study population consisted of outpatients visiting health care centres for uncomplicated falciparum malaria-like symptoms. Patients were enrolled if they meet the following selection criteria: 1) at least two years old; 2) fever with axillary temperature $>$ or $=37.5^{\circ}$ C; 3) P. falciparum mono-infection with a parasitaemia from 2,000 to 200,000/ $\mu$ l of blood, in Cameroon and Côte d'Ivoire, and from 1,000 to $100,000 / \mu$ of blood in Sénégal; 4) provision of the written and informed consent by the patient or his legal guardian for children; 5) no history of previous serious side-effects to the drugs used in the trial; 6) no evidence of a concomitant febrile illness; 7) no danger signs or evidence of severe malaria;
8) no treatment with 4-amino quinoleines, sulphadoxine-pyrimethamine, mefloquine or halofantrine in the previous seven days, or with quinine, artemisinin or cyclins in the previous three days, 9) no pregnancy or nursing; 10) no ongoing anti-malarial treatment.

The number of patients to be enrolled was determined by Epi Info 2000 software. The estimated expected recovery rate with AL was $98 \%$, with a maximum acceptable difference of $5 \%$ to conclude that DP was noninferior and a power of $85 \%$. The minimum number of people to be included in each arm was calculated from these assumptions to be 180 patients. Assuming a 5\% loss to follow up the overall final target sample size of 380 participants was estimated.

\section{Study design and treatment}

This study was a randomized, controlled and open therapeutical trial of DP against AL as the reference treatment. The three criteria of judgment were clinical efficacy, biological efficacy and tolerance. The study protocol was first approved in each country by the National Ethical Committee according to protocols and standards operating procedures of Good Clinical Practices of the ICH harmonized Triplicate Guide Lines for Good Clinical Practice made in 1996 and the Helsinki Declaration on human being research. This approval was critical for the study start.

For each patient who met inclusion criteria, the protocol was read and explained to him/her or the legal guardian (for children) who in case of acceptance had to sign the written informed sheet afterward to authorize recruitment of the child in the study. The patient or guardian was given a copy of the informed consent and patient information sheet. For patients who gave their consent, baseline examinations and laboratory investigations were carried immediately free of charge. Those of patients who met inclusion criteria at baseline (day 1) were randomly assigned to one of the two treatment groups following a randomization list. In each study site computer generated randomization codes were prepared by an independent individual. These codes were enclosed in sequentially numbered opaque sealed envelopes, each of which contained the treatment allocation. The envelopes were assigned in sequential order to participants after inclusion.

Treatments in the two groups were allocated according to body weight. First patient group was allocated dihydroartemisinin-piperaquine (DP) (Duocotecxin ${ }^{\circledR}$ ) (Beijing Holley-Cotec Pharmaceutical Co. Ltd, China) once daily for three consecutive days. Each tablet of DP contains $40 \mathrm{mg}$ of dihydroartemisinin and $320 \mathrm{mg}$ of piperaquine. DP treatments varied as follows: patients between $5-9 \mathrm{~kg}$ received half a tablet per dose, those between 10-14 kg 3/4 tablet, 15-19 $\mathrm{kg} 1$ tablet per dose, 
20-24 kg 1 tablet $+1 / 4$ per dose, $25-29 \mathrm{~kg} 1$ tablet $+1 / 2$ per dose, 30-34 kg 1 tablet $+3 / 4$ per dose, $35-39 \mathrm{~kg} 2$ tablets per dose, $40-44 \mathrm{~kg} 2$ tablets $+1 / 4$ per dose, 45 $49 \mathrm{~kg} 2$ tablets $+1 / 2$ per dose and patients $\geq 50 \mathrm{~kg} 3$ tablets per dose. The second patient group was allocated artemether-lumefantrine (AL) $\left(\right.$ Coartem $\left.^{\circledR}\right)$ tablets (Novartis Pharma, Switzerland), each tablet containing $20 \mathrm{mg}$ artemether and $120 \mathrm{mg}$ lumefantrine. Patients received treatment dose according to the following scheme: i.e. patients between $5-14 \mathrm{~kg}$ received one tablet per dose, those between $15-24 \mathrm{~kg}$ two tablets, those between $25-34 \mathrm{~kg}$ received three tablets per dose and those with body weight $\geq$ to $35 \mathrm{~kg}$ received four tablets per dose. Doses were given at T0h, T8h, T24h, T36h, $\mathrm{T} 48 \mathrm{~h}$ and T60h. All treatments were given under direct supervision of the study co-investigators. If the patient vomited within thirty minutes after drug administration, the whole dose was re-administered. If the vomiting persisted, the patient was excluded from the study and referred to the health centre doctor for management according to the current national policy. The dose could not be administered again if vomiting occurred more than 60 minutes after administration.

After inclusion, the patient had to come to follow-up visits during which clinical and physical examinations as well as laboratory investigations were carried out by the physician and laboratory technicians.

\section{Efficacy and tolerance}

Clinical and biological efficacies were assessed using the WHO in vivo test with a follow-up period of 28 days $[14,15]$. At enrollment (day 1 ) as well as follow-up visits, a full clinical and physical examination was performed; data were recorded in a case report form. Laboratory investigations for P. falciparum stages and loads were also carried out at baseline and follow-up visits by means of finger pricking for thin and thick smears. Follow-up visits were on day 2 , day 3 , day 4 , day 7 , day 14 , day 21 and day 28 after the first anti-malarial drug had been swallowed. Finger prick blood samples were collected for microscopy. Blood samples were also collected for P. falciparum molecular biology analysis at baseline and then after day 7 in case of positive parasitaemia to check if parasitaemia recurred in patients. Response to treatment was measured and defined according to WHO guidelines [15]. Other blood samples were collected by venipuncture at baseline and day 4 for the measurement of haemoglobin levels and biochemical parameters, such as bilirubin, creatinin and transaminases (AST, ALT). Patients developing danger signs during follow-up visits were withdrawn from the study, referred to the appropriate hospital ward for care and medication. Other patients showing complications or treatment failure were also referred for appropriate care and medication but remained in the study for the follow-up. Adverse events (AE) were recorded on the case report forms and their gravity was graded as mild, moderate or severe. An AE was defined as an unfavourable and unintended symptom, sign or disease.

\section{Parasite clearance and gametocyte dynamics}

Plasmodium falciparum parasite clearance was assessed microscopically. For this, Giemsa-stained thick and thin blood smears were prepared according to WHO guidelines [15]. Two independent, experienced microscopists, examined the smears for the presence and quantification of parasites species.

\section{Genotyping}

In order to distinguish re-infection (RI) from recrudescence (RE), merozoite surface protein 1 and 2 ( $m s p 1$ and $m s p 2$ ) genotyping was performed as described by Faye [16] on dried blood spots collected at the patient enrollment (day 1) and at the time when parasitaemia reccurrence was noticed. Blood spots were collected on Whatman filter paper (Whatman International Ltd. Maidstone, UK) and air-dried at room temperature for PCR analysis. DNAwas extracted using the methanol method [17]. Molecular biology analysis was performed at the Parasitology Laboratory of Cheikh Anta Diop University (Dakar, Sénégal).

\section{End points}

The primary end point was the recovery rate by 28 , defined as the percentage of patients who had an adequate clinical and parasitological response (ACPR) after follow-up for 28 days. Efficacy was evaluated using a modified intention to treat analysis, which included the 379 patients, randomized and not lost to follow-up. The secondary end points were the incidence of early clinical failure (ECF), late parasitological failure (LPF), late clinical failure (LCF), change in gametocyte carrier status, fever and parasites clearance and adverse clinical and laboratory events.

\section{Statistical analysis}

All data were recorded and checked using Epi data version 3.1 and analysed with SPSS for windows (version 12.0). Patient's characteristics of the two groups at inclusion were compared using Pearson's Chi-2 test, independent samples t test or Mann-Whitney test. The modified intention to treat analysis was performed using Kaplan-Meier survival analysis with log rank testing the treatment failures distribution function. The cases of protocol violation and withdrawn consent were censored at the time they left the study. The distributions of fever and parasite clearance were compared using Pearson's Chi-2 test. Differences of haemoglobin and biochemical 
parameters values within individuals between D1 and D4 were computed. Changes in haemoglobin concentrations and in biochemical parameters were compared using the paired $t$ test. The level of significance for statistical tests was set at 0.05 .

\section{Results}

\section{Global distribution and baseline characteristics of} patients in the study

A total of 384 patients were included in the study. 197 patients were randomized to DP and 187 to AL. In the DP group, six patients were excluded after enrollment. Of these, four patients were lost during follow-up and one case of protocol violation (self-medication with another anti-malarial drug) and one case of withdrawal of consent were noticed. In the AL group, four patients were excluded during the follow-up: one patient was lost sight. There were one case of protocol violation and two cases of withdrawal of the consent.

Finally, 191 patients and 183 patients were successfully followed up, respectively in DP and AL groups (Figure 1). Baseline characteristics of patients receiving either AL or DP are presented in Table 1 and Table 2. There were no statistically significant differences in distribution by sex or age band. Average temperatures in the two treatment groups were not statistically different. There were also no statistically significant differences between any of the laboratory parameters. Both groups were, therefore, considered statistically equivalent at inclusion.

\section{Clinical and parasitological outcomes}

Kaplan-Meier estimates of recovery rates unadjusted by genotyping were $99.0 \%$ in the DP group and $97.3 \%$ in the AL group. There was no statistical difference between the two groups $(\mathrm{p}=0.230)$. These recovery rates adjusted by genotyping, $99.5 \%$ in the DP group and $98.9 \%$ in the AL group, were not statistically different $(p=0.538)$. There was no significant study site effect on these estimates.

No Early Therapeutic Failure (ETF) was observed. At day 28, 2 patients in the DP group and 5 in AL group had recurrent parasitaemia with $P$ falciparum. In the DP group, after PCR genotyping, one of the two recurrences was classified as a new infection and the other as recrudescence. In AL group, two recurrences were classified after correction by PCR as recrudescence. All cases of recrudescence were classified as Late Parasitological Failure (LPF).

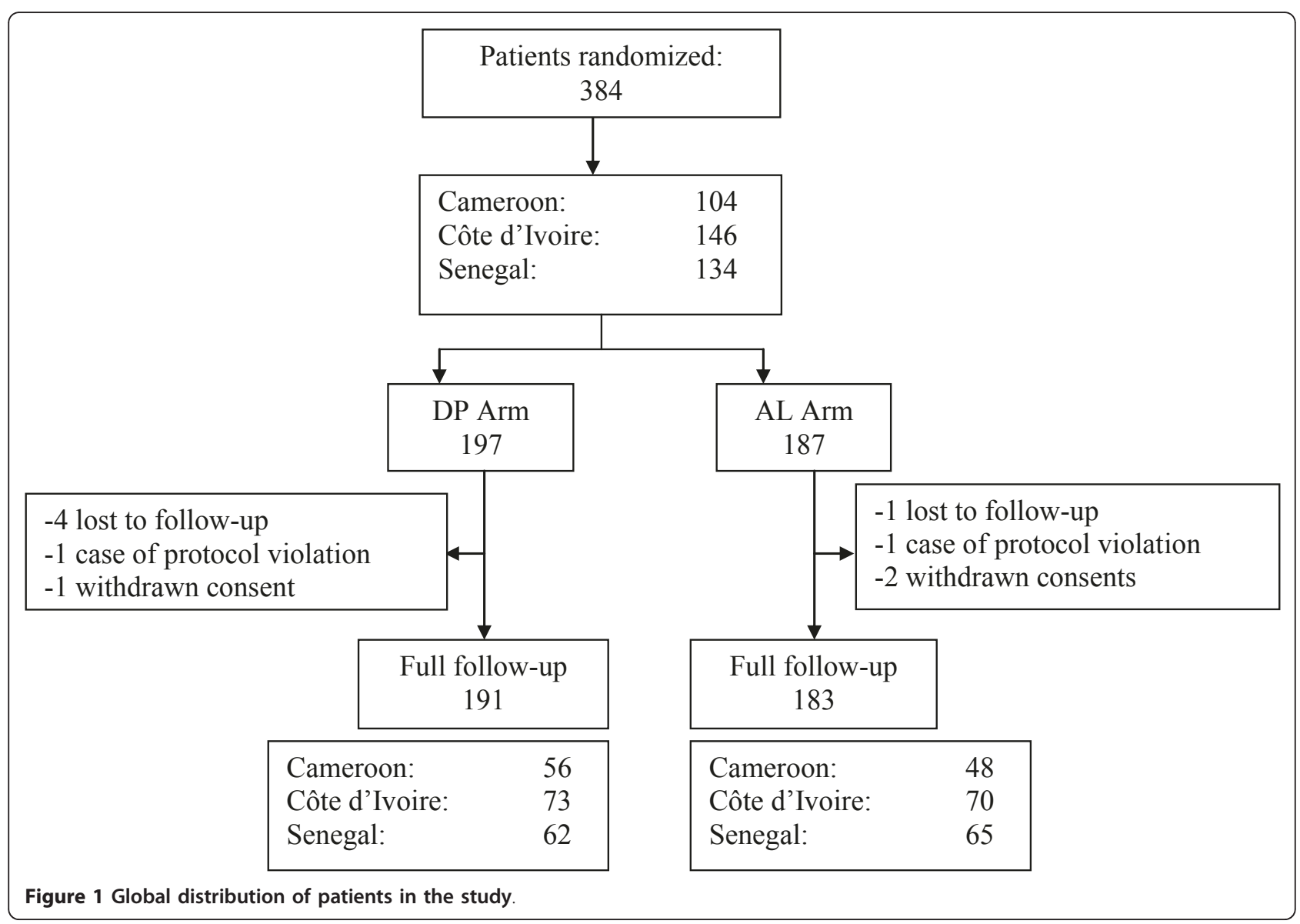


Table 1 Comparison of the two treatment groups at inclusion

\begin{tabular}{|c|c|c|c|}
\hline & DP & $\mathrm{AL}$ & $\mathrm{p}^{*}$ \\
\hline Numbers & 197 & 187 & \\
\hline \multicolumn{4}{|l|}{$\overline{\text { Sex }}$} \\
\hline M, n (\%) & $97(49.2)$ & $93(47.2)$ & $0.923^{* *}$ \\
\hline $\mathrm{F}, \mathrm{n}(\%)$ & $100(50.8)$ & $94(52.8)$ & \\
\hline Mean age (SD) years & $15.64(14.22)$ & $13.48(12.64)$ & 0.117 \\
\hline$[2-5[, n(\%)$ & $34(17.3)$ & $44(23.7)$ & \\
\hline$[5-15[, n(\%)$ & $92(46.7)$ & $83(44.6)$ & \\
\hline [15-77], n (\%) & $71(36.0)$ & $59(31.7)$ & \\
\hline Mean temperature (SD) ${ }^{\circ} \mathrm{C}$ & $38.42(0.64)$ & $38.43(0.73)$ & 0.887 \\
\hline$[37.5-38.5[, \mathrm{n}(\%)$ & $114(57.9)$ & $113(60.4)$ & \\
\hline [38.5 - 41.0], n (\%) & $83(42.1)$ & $74(39.6)$ & \\
\hline Median parasitaemia tpz/ $\mu \mathrm{l}$ & 10840 & 16000 & $0.546 \S$ \\
\hline Min - Max & $1000-200000$ & $1000-200000$ & \\
\hline Gametocyte carrier rate, n (\%) & $8(4.1)$ & $6(3.2)$ & $0.649^{* *}$ \\
\hline Mean AST (SD) IU/I & $26.64(15.53)$ & $31.1(29.08)$ & 0.065 \\
\hline Mean ALT (SD) IU/I & $18.71(12.71)$ & $24.06(36.63)$ & 0.061 \\
\hline Mean creatinin (SD) mg/l & $6.79(2.41)$ & $6.76(2.47)$ & 0.915 \\
\hline Mean bilirubin (SD) mg/l & $10.43(7.21)$ & $11.4(8.65)$ & 0.236 \\
\hline Mean haemoglobin (SD) g/dl & $10.79(2.06)$ & $10.67(2.12)$ & 0.575 \\
\hline
\end{tabular}

*Independent samples t test **Pearson's Chi-2 test §Mann-Whitney test

In the two groups, almost $94 \%$ of patients had cleared parasitaemia within 48 hours after enrollment; there was no statistical difference between the two groups ( $\mathrm{p}=$ 0.866) (Figure 2). Fever clearance was equally high: at day 3 almost all patients were apyretic, with no difference between the 2 groups $(p=0.94)$ (Figure 3$)$. At inclusion, there were only eight gametocyte carriers in the DP group and six in the AL group. The number of gametocyte carriers decreased to one on day 3 in the DP group and to two in the AL group. From day 4, there were no more gametocyte carriers in both groups.

\section{Adverse events}

Among the 384 patients followed, 35 (23 in DP group and 12 in AL group) had adverse events (9.1\%). There

Table 2 Clinical signs of patients at inclusion

\begin{tabular}{lccc}
\hline & DP (n, \%) & AL (n, \%) & Total n (\%) \\
\hline Fever & $197(100 \%)$ & $187(100 \%)$ & $384(100)$ \\
Headache & $167(84.8 \%)$ & $153(81.8 \%)$ & $320(83.3)$ \\
Asthenia & $153(81.8 \%)$ & $106(56.7 \%)$ & $223(58.1)$ \\
Anorexia & $103(52.3 \%)$ & $95(50.8 \%)$ & $198(51.6)$ \\
Shiver & $93(47.2 \%)$ & $80(42.8 \%)$ & $173(45.1)$ \\
Joint pain & $77(39.1 \%)$ & $75(40.1 \%)$ & $152(39.6)$ \\
Abdominal pain & $35(17.8 \%)$ & $43(23 \%)$ & $78(20.3)$ \\
Insomnia & $18(9.1 \%)$ & $23(12.3 \%)$ & $41(10.7)$ \\
Dizziness & $18(9.1 \%)$ & $13(6.9 \%)$ & $31(8.1)$ \\
\hline
\end{tabular}

was no severe adverse event in the two groups and no treatment was interrupted due to adverse events. Most of adverse events were of moderate severity. The most commonly reported adverse events in both treatment groups were abdominal pain, dizziness, diarrhoea, vomiting, pruritus and nausea (Table 3). Adverse events were not significantly different between the two treatment groups.

A decrease of haemoglobin values was observed from the beginning of treatment to day 4 . This decrease was more important in AL group $(0.36 \mathrm{~g} / \mathrm{dl})$ than DP group $(0.14 / \mathrm{g} / \mathrm{dl})$. The decrease was significant in AL group ( $\mathrm{p}$ $=0.001)$ but not in DP group $(\mathrm{p}=0.221)$. In DP group from the beginning of the treatment to day 4, there was a decrease of the mean of AST and a small increase of ALT mean, while in the AL group, AST and ALT means increased. However, these variations were not significantly different. The decrease of the mean of creatinin from the beginning of the treatment to day 4 was not significant in the DP group but was significant in the AL group. In the two groups, the bilirubin decrease was significant (Table 4).

\section{Discussion}

In this study, the efficacy and tolerability of two ACT formulations have been compared in a randomized trial of patients with uncomplicated malaria and followed up for 28 days. Both treatments were highly efficient and 

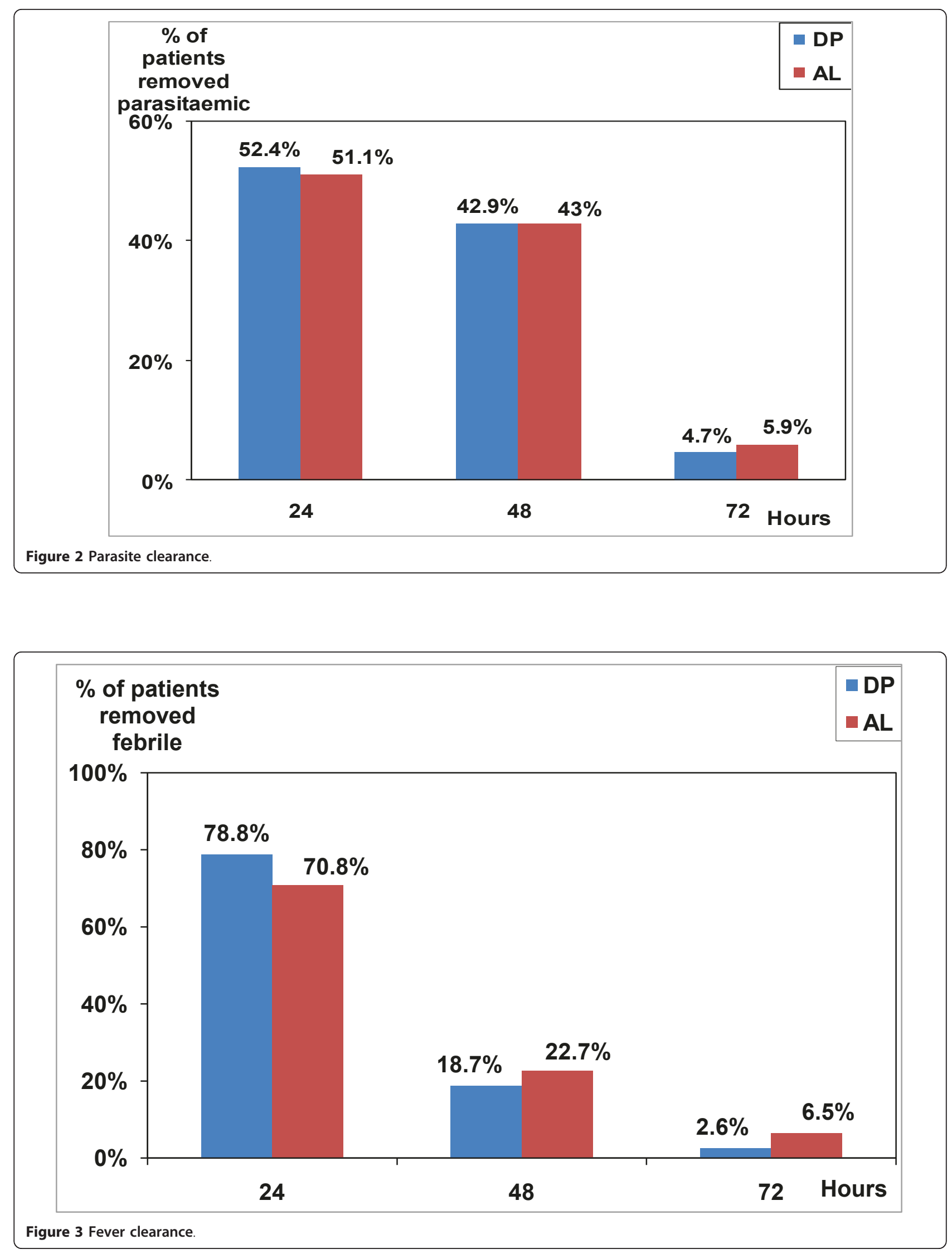
Table 3 Adverse events recorded during the study

\begin{tabular}{lccc}
\hline Adverse events & DP $(\mathbf{n}, \%)$ & AL $(\mathbf{n}, \%)$ & Total $(\mathbf{n}, \%)$ \\
\hline Abdominal pain & $9(4.6)$ & $9(4.8)$ & $18(4.7)$ \\
Dizziness & $5(2.5)$ & $2(1.1)$ & $7(1.8)$ \\
Diarrhoea & $4(2)$ & $2(1.1)$ & $6(1.6)$ \\
Vomiting & $4(2)$ & $0(0)$ & $4(1)$ \\
Pruritus & $3(1.5)$ & $1(0.5)$ & $4(1)$ \\
Nausea & $2(1)$ & $2(1.1)$ & $4(1)$ \\
Face oedema & $1(0.5)$ & $0(0)$ & $1(0.3)$ \\
Sleepiness & $1(0.5)$ & $1(0)$ & $1(0.3)$ \\
Insomnia & $0(0)$ & $1(0.5)$ & $1(0.3)$ \\
\hline
\end{tabular}

Table 4 Evolution of biological parameters in the two groups

\begin{tabular}{|c|c|c|c|c|c|c|c|c|}
\hline & \multicolumn{4}{|c|}{ DP } & \multicolumn{4}{|c|}{$A L$} \\
\hline & D1 & D4 & D1-D4 & $\mathrm{p}^{*}$ & D1 & D4 & D1-D4 & $\mathrm{p}^{*}$ \\
\hline $\begin{array}{l}\text { AST (IU/I) } \\
\text { (SD) }\end{array}$ & $\begin{array}{c}26.48 \\
(15.17)\end{array}$ & $\begin{array}{c}24.79 \\
(12.10)\end{array}$ & $\begin{array}{c}1.70 \\
(17.51)\end{array}$ & 0.190 & $\begin{array}{c}29.62 \\
(26.14)\end{array}$ & $\begin{array}{c}38.24 \\
(131.0)\end{array}$ & $\begin{array}{l}-8.63 \\
(132.6)\end{array}$ & 0.389 \\
\hline $\begin{array}{l}\text { ALT (IU/I) } \\
\text { (SD) }\end{array}$ & $\begin{array}{c}18.91 \\
(12.71)\end{array}$ & $\begin{array}{c}19.73 \\
(12.91)\end{array}$ & $\begin{array}{l}-0.82 \\
(13.77)\end{array}$ & 0.418 & $\begin{array}{l}24.20 \\
(37.46)\end{array}$ & $\begin{array}{c}34.19 \\
(138.47)\end{array}$ & $\begin{array}{l}-9.99 \\
(140.5)\end{array}$ & 0.347 \\
\hline $\begin{array}{l}\text { Creatinin (mg/l) } \\
\text { (SD) }\end{array}$ & $\begin{array}{c}6.81 \\
(2.46)\end{array}$ & $\begin{array}{c}6.70 \\
(2.41)\end{array}$ & $\begin{array}{c}0.11 \\
(1.52)\end{array}$ & 0.317 & $\begin{array}{c}6.86 \\
(2.45)\end{array}$ & $\begin{array}{l}6.51 \\
(2.51)\end{array}$ & $\begin{array}{l}0.35 \\
(1.9)\end{array}$ & 0.001 \\
\hline $\begin{array}{l}\text { Bilirubin (mg/l) } \\
\text { (SD) }\end{array}$ & $\begin{array}{l}10.49 \\
(7.39)\end{array}$ & $\begin{array}{c}6.31 \\
(2.99)\end{array}$ & $\begin{array}{l}4.18 \\
(7.42)\end{array}$ & $<0.001$ & $\begin{array}{l}11.19 \\
(8.51)\end{array}$ & $\begin{array}{c}6.27 \\
(3.30)\end{array}$ & $\begin{array}{c}4.92 \\
(8.57)\end{array}$ & $<0.001$ \\
\hline $\begin{array}{l}\text { Haemoglobin (g/dl) } \\
\text { (SD) }\end{array}$ & $\begin{array}{l}10.80 \\
(2.07)\end{array}$ & $\begin{array}{l}10.66 \\
(2.18)\end{array}$ & $\begin{array}{c}0.14 \\
(1.56)\end{array}$ & 0.221 & $\begin{array}{l}10.75 \\
(2.07)\end{array}$ & $\begin{array}{l}10.39 \\
(2.08)\end{array}$ & $\begin{array}{c}0.36 \\
(1.48)\end{array}$ & 0.001 \\
\hline
\end{tabular}

*paired t test

well tolerated as previously described [1]. Moreover, several studies with a follow-up of 42 days have been carried out about the efficacy and tolerability of DP and AL and all showed very good results [18-21]. The rate of true recrudescence with $P$. falciparum was less than $5 \%$ in both treatment groups, and most P. falciparum recurrences were caused by reinfection. These outcomes are in accordance with previous efficacy studies of AL $[13,23]$ and DP $[7,9,11,24,25]$.

The fact that there was more reinfection than recrudescence shows that malaria transmission is very high in these areas (especially in Côte d'Ivoire and Cameroon). Thus, even if anti-malarial drugs are effective and welltolerated, it is very important to continue to use prevention tools, such as long-lasting insecticide-treated nets to fight malaria.

The haemoglobin rate decrease was significant in the AL group, but not in the DP group. This result suggests that convalescence is obtained faster in the DP group. However, as these data were obtained only four days after enrollment, it would be more significant to assess the haemoglobin rate decrease over 28 days. As in several studies which reported good tolerance of DP $[25,26]$ and AL [26], there were no serious adverse events during the follow-up in the assessment.
In conclusion, DP presented good and equivalent efficacy and tolerability profile as AL. Therefore, DP is a good alternative for the first-line treatment of uncomplicated $P$. falciparum malaria in endemic regions. Its once daily dose is even more a significant advantage because it could contribute to improve the patient's treatment compliance.

\section{List of abbreviations}

DP: combination Dihydroartemisinin - Piperaquine phosphate; AL: Artemether - Lumefantrine.

\section{Acknowledgements and funding}

We are grateful for the hospitality and generous collaboration of the staff of Health centers where the study took place. We thank also the patients and parents of the patients included in this study for their collaboration.

The study received financial support from Beijing Holley-Cotec Pharmaceutical Co., Ltd.

Dihydroartemisinin-piperaquine study drugs were provided free of charge by Beijing Holley-Cotec Pharmaceutical Co., Ltd.

The funders had no involvement in the study design, data collection, data analysis, data interpretation, in the writing of the manuscript, or in the decision to submit it for publication.

\section{Author details}

${ }^{1}$ Department of Parasitology and Mycology, Faculty of Pharmaceutical and Biological Sciences, Abidjan, Côte d'Ivoire. ${ }^{2}$ Parasitology and Mycology Laboratory, Faculty of Medecine, UCAD, Dakar, Sénégal. ${ }^{3}$ Parasitology Laboratory, Faculty of Medecine and Pharmaceutical Sciences of Douala, Yaoundé, Cameroon. ${ }^{4}$ Departement of Biostatistic and Public Health, Faculty of Pharmaceutical and Biological Sciences, Abidjan, Côte d'Ivoire. ${ }^{5}$ Projet 
RETROCl, Abidjan, Côte d'Ivoire. ' $M a l a r i a$ Research and Control Center, National Institute of Public Health, Abidjan, Côte d'Ivoire. ${ }^{7}$ University Hospital Center, Yaoundé, Cameroon.

\section{Authors' contributions}

OF, SE, KM supervised the clinical studies. KM was the principal investigator $\mathrm{SAO}, \mathrm{WY}$ and KM analysed the data. All authors read and approved the final manuscript.

\section{Competing interests}

The authors declare that they have no competing interests.

Received: 19 August 2010 Accepted: 20 July 2011

Published: 20 July 2011

\section{References}

1. Mens PF, Sawa P, van Amsterdam SM, Versteeg I, Omar SA, Schallig HD, Kager PA: A randomized trial to monitor the efficacy and effectiveness by QT-NASBA of artemether-lumefantrine versus dihydroartemisinin-piperaquine for treatment and transmission control of uncomplicated Plasmodium falciparum malaria in western Kenya. Malar J 2008, 7:237.

2. World Health Organization: World malaria report 2008 Geneva; 2008

3. Adjuik M, Babiker A, Garner P, Olliaro P, Taylor W, White N: Artesunate combinations for treatment of malaria: meta-analysis. Lancet 2004, 363:9-17.

4. Ashley EA, Stepniewska K, Lindegardh N, Annerberg A, Kham A, Brockman A, Singhasivanon P. White NJ, Nosten F: How much fat is necessary to optimize lumefantrine oral bioavailability? Trop Med Int Health 2007, 12:195-200.

5. Bousema JT, Schneider P, Gouagna LC, Drakeley CJ, Tostmann A, Houben R, Githure Jl, Ord R, Sutherland CJ, Omar SA, Sauerwein RW: Moderate effect of artemisinin-based combination therapy on transmission of Plasmodium falciparum. J Infect Dis 2006, 193:1151-1159.

6. Broek I van den, Kitz C, Al Attas S, Libama F, Balasegaram M, Guthmann JP. Efficacy of three artemisinin combination therapies for the treatment of uncomplicated Plasmodium falciparum malaria in the Republic of Congo. Malar J 2006, 5:113.

7. Denis MB, Davis TM, Hewitt S, Incardona S, Nimol K, Fandeur T, Poravuth $Y$, Lim C, Socheat D: Efficacy and safety of dihydroartemisinin-piperaquine (Artekin) in Cambodian children and adults with uncomplicated falciparum malaria. Clin Infect Dis 2002, 35:1469-1476.

8. Karunajeewa H, Lim C, Hung TY, llett KF, Denis MB, Socheat D, Davis TM: Safety evaluation of fixed combination piperaquine plus dihydroartemisinin (ArtekinH) in Cambodian children and adults with malaria. Br J Clin Pharmacol 2004, 57:93-99.

9. Tran TH, Dolecek C, Pham PM, Nguyen TD, Nguyen T, Le HT, Dong TH, Tran TT, Stepniewska K, White NJ, Farrar J: Dihydroartemisinin-piperaquine against multidrug resistant Plasmodium falciparum malaria in Vietnam: randomised clinical trial. Lancet 2004, 363:18-22

10. Mayxay $M$, Thongpraseuth $V$, Khanthavong $M$, Lindegårdh $N$, Barends $M$ Keola S, Pongvongsa T, Phompida S, Phetsouvanh R, Stepniewska K, White NJ, Newton PN: An open, randomized comparison of artesunate plus mefloquine vs dihydroartemisinin-piperaquine for the treatment of uncomplicated Plasmodium falciparum malaria in the Lao People's Democratic Republic (Laos). Trop Med Int Health 2006, 11:1157-1165.

11. Smithuis F, Kyaw MK, Phe O, Aye KZ, Htet L, Barends M, Lindegardh N, Singtoroj T, Ashley E, Lwin S, Stepniewska K, White NJ: Efficacy and effectiveness of dihydroartemisinin-piperaquine versus artesunatemefloquine in falciparum malaria: an open label randomized comparison. Lancet 2006, 367:2075-2085.

12. Janssens B, van Herp M, Goubert L, Chan S, Uong S, Nong S, Socheat D, Brockman A, Ashley EA, Van Damme W: A randomized open study to assess the efficacy and tolerability of dihydroartemisinin-piperaquine for the treatment of uncomplicated falciparum malaria in Cambodia. Trop Med Int Health 2007, 12:251-259.

13. Piola P, Fogg C, Bajunirwe F, Biraro S, Grandesso F, Ruzagira E, Babigumira J, Kigozi I, Kiguli J, Kyomuhendo J, Ferradini L, Taylor W, Checchi F, Guthmann JP: Supervised versus unsupervised intake of six-dose artemetherlumefantrine for treatment of acute, uncomplicated
Plasmodium falciparum malaria in Mbarara, Uganda: a randomised trial. Lancet 2005, 365:1467-1473.

14. Fanello Cl, Karema C, van Doren W, van Overmeir C, Ngamije D, D'Alessandro $U$ : A randomised trial to assess the safety and efficacy of artemether-lumefantrine $\left(\right.$ Coartem $\left.^{\circledast}\right)$ for the treatment of uncomplicated Plasmodium falciparum malaria in Rwanda. Trans $R$ Soc Trop Med Hyg 2007, 101:344-350.

15. World Health Organization: Assessment and monitoring of antimalarial drug efficacy for the treatment of uncomplicated falciparum malaria Geneva; 2003.

16. Faye B, Ndiaye JL, Tine R, Sylla K, Gueye A, Lo A, Gaye O: A randomized trial of artesunate mefloquine versus artemether lumefantrine for the treatment of uncomplicated Plasmodium falciparum malaria in Senegalese children. Am J Trop Med Hyg 2010, 82:140-144

17. Dicko A, Sagara I, Djimdé AA, Touré SO, Traore M, Dama S, Diallo A Barry A, Dicko M, Coulibaly OM, Rogier C, de Sousa A, Doumbo OK: Molecular markers of resistance to sulphadoxine-pyrimethamine one year after implementation of intermittent preventive treatment of malaria in infants in Mali. Malar J 2010, 9:9.

18. Ashley EA, McGready R, Hutagalung R, Phaiphun L, Slight T, Proux S, Thwai KL, Barends M, Looareesuwan S, White NJ, Nosten F: A randomized controlled study of a simple, once-daily regimen of dihydroartemisininpiperaquine for the treatment of uncomplicated, multidrug-resistant falciparum malaria. Clin Infect Dis 2005, 41:425-432.

19. Kamya MR, Yeka A, Bukirwa H, Lugemwa M, Rwakimari JB, Staedke SG, Talisuna AO, Greenhouse B, Nosten F, Rosenthal PJ, Wabwire-Mangen F, Dorsey G: Artemether-lumefantrine versus dihydroartemisininpiperaquine for treatment of malaria: a randomized trial. PLOS Clin Trials 2007, 2:20

20. Karema C, Fanello Cl, van Overmeir C, van Geertruyden J-P, van Doren W Ngamije D, D'Alessandro U: Safety and efficacy of dihydroartemisinin/ piperaquine (Artekin ${ }^{\circledast}$ ) for the treatment of uncomplicated Plasmodium falciparum malaria in Rwandan children. Trans R Soc Trop Med Hyg 2006, 101:1105-1111

21. Osorio L, Gonzalez IJ, Olliaro P, Taylor WR: Artemisinin-based combination therapy for uncomplicated Plasmodium falciparum malaria in Colombia. Malar J 2007, 6:25.

22. Ratcliff A, Siswantoro H, Kenangalem E, Maristela R, Wuwung MR, Laihad F, EbsworthP PE, Anstey MN, Tjitra E, Price NR: Two fixed-dose artemisinin combinations for drug-resistant falciparum and vivax malaria in Papua, Indonesia: an open-label randomised comparison. Lancet 2007, 369:757-765

23. van Vugt $M$, Looareesuwan $S$, Wilairatana $P, M c G r e a d y ~ R$, Villegas $L$, Gathmann I, Mull R, Brockman A, White NJ, Nosten F: Artemetherlumefantrine for the treatment of multidrug-resistant falciparum malaria. Trans R Soc Trop Med Hyg 2000, 94:545-548.

24. Ashley EA, Krudsood S, Phaiphun L, Srivilairit S, McGready R, Leowattana W, Hutagalung R, Wilairatana P, Brockman A, Looareesuwan S, Nosten F, White NJ: Randomized, controlled dose-optimization studies of dihydroartemisinin-piperaquine for the treatment of uncomplicated multidrug-resistant falciparum malaria in Thailand. J Infect Dis 2004, 190:1773-1782.

25. Myint HY, Ashley EA, Day NP, Nosten F, White NJ: Efficacy and safety of dihydroartemisinin-piperaquine. Trans R Soc Trop Med Hyg 2007, 101:858-866

26. Wang SQ, Christophel E, Lin SG, Meng F, Hu XM, Wang GZ, Liu J, Zeng LH: Efficacy of dihydroartemisinin-piperaquine and artemether-lumefantrine in the treatment of uncomplicated falciparum malaria in Hainan, China. Zhongguo Ji Sheng Chong Xue Yu Ji Sheng Chong Bing Za Zhi 2008 , 26.50-52.

doi:10.1186/1475-2875-10-198

Cite this article as: Yavo et al:: Multicentric assessment of the efficacy and tolerability of dihydroartemisinin-piperaquine compared to artemether-lumefantrine in the treatment of uncomplicated Plasmodium falciparum malaria in sub-Saharan Africa. Malaria Journal 2011 10:198. 